\title{
El concepto Indice Delta en la determinación del valor funcional de los riñones.
}

Por el Dr. LUIS PRADO BARRIENTOS.

(Del Curso de Aplicacion del Instituto Oswaldo Cruz).

Antes de entrar en el asunto que motiva esta exposición, expreso mis agradecimientos a los señores profesores, Dr. GOMES de FARIA y CARNEIRO FELIPPE, a cuyos estímulos se deben la génesis y la construcción de un pequeño aparato que determina mecanicamente lo que ha venido en llamarse Indice Delta.

Entre las multiples investigaciones encaminadas a ave riguar el valor funcional de los riñones en un momento dado, ninguna de ellas tiene actualmente mas aplicacion-en patologia renal-que la determinacion de la Constante ureo-secretoria de AMBARD.

La funcion biológica de eliminacion ureica está regida por leyes deducidas experimentalmente por L. AMBARD.-Esas leyes se traducen en formulas matemáticas, que es preciso resolverlas en cada caso, en vista de los datos proporcionados por el laboratorio.

La Constante ureo-secretoria, en culanto se la considera como problema sometido a calculos numéricos, presenta dos inconvenientes, que se ha tratado de allanar.

Primeiro.-Las cifras que expresan la Constante ureo-secretoria, siendo números fraccionarios (desde 0,015 aproximadamente, hasta 0,800 , o mas) no presentan al clinico la facil comprension del valor funcional de los riñones, en lo que se relaciona con la escresion ureica.

Segundo.-No todos los medicos estan familiarizados con la resolucion de problemas numéricos, ni a todos los tecnicos de laboratorio les es dado substraer el tiempo dedicado á cuidadosas investigaciones de las casi siempre dependen el diagnóstico, el prognóstico y el tratamiento de las enfermedades.

Para evitat el primer inconveniente, CARRION y HALLION, ya propucieron expresar el valor de la excresion ureica en porcentage, basandose en que la cifra normal de la Constante ureo-secretoria 0,070 , corresponde a 100.

C. Mc LEAN, en su trabajo "The numerical laws governing the rate excretion of urea and chlorides in man" (Journal of exp. Med.-1925-V. 22 - pgs. 212-236), fundado en gran número de observaciones estudiadas cuidadosamente y empleando en las dosificaciones de urea el xantidrol, primeiro estabelece que, la Constante ureo-secretoria normal es: 0,080 y no 1),070, como hasta entonces se aceptaba; segundo propone que, para expresar los cambios de valor de la Constante, de una manera matemáticamente correcta, basada en la escala 100 , se use de una fórmula adoptada a las 
leyes de AMBARD, que expresaria el Indice de excresion ureica. El numero 100 o standard normal, corresponderia a la normal 0,080 de la Constante ureo-secretoria de AMBARD. Las variaciones serian expresadas en terminos normales formando la serie sucesiva de los números de orden. Ver el cuadro de la pagina n. 16.

El trabajo que hoy presento, lleva el mismo fin, es decir: apreciar en forma simple y de facil comprensión el valor de excresión ureica, representando ese valor et porcentage de trabajo renal para la excresión de la urea, que viene a ser el desvio del Indice normal de Mc LEAN, a lo que se ha denominado Indice Delta.

El segundo inconveniente ha sido allanado en el sentido de determinar los valores de la Constante ureo-sesretoria, por dispositivos ó aparatos que dispensan cálculos numéricos.

a) -En primer término debemos citar el "Abaque de ANDRE" GUERBET» (Journal de Pharmacie et Chimie.-tomo 30 1924-pag. 68), que reduce el problema numérico de la Constante DE AMBARD a simples manipulaciones de escalas nomográficas, yá estudiadas por MAURICE OCAGNE.

b) - El abaco de BERGEROL y LAZARD, en que hay necesidad de calcular el debit ureico.-

c) -El Ureografo de HAMEL, aparato que he tenido la oportunidad de conocer ultimamente, despues de concluido este trabajo. Es un aparato muy ingenioso de mecanismo interno, en que el problema se reduce á anotar los datos proporcionados por el laboratorio, obteniendose la Constante automáticamente.

d) -El Aparato destinado a la determinación del Indice Delta, que tambien determina, y simultaneamente, el Indice de Mc. LEAN y la Constante ureo secretoria de AMBARD, y para cuya construccion se tuvo en cuenta el ábaco de ANDRE' GUERBET.

Constante de AMBARD - Si se examina el cuadro comparativo de la pagina $n .16$ se verá que en las variaciones de $K$ (constante ureo-secretoria de AMBARD), a partir del valor normal, que es, 0,080 las cifras inferiores a ese valor, indican un exeso de trabajo renal para la eliminacion ureica (caso de degeneración amiloidea del riñon, nefritis hidropígena, diañetis, fiebre amarilla, etc.). Inversamente las cifras superiores a 0,080 indican una insuficiencia renal para esa funcion.

El Indice de Mc LEAN, fundado en las leyes de AMBARD, concidera como valor normal 100. Las cifras superiores a 100 indican un exeso de funcion, pudiendo llegar hasta 400,500 , etc.

Las cifras inferiores a 100 indican deficiencia renal, pudiendo bajar teoricamente hasta la unidad.

Tanto en la escala de numeros fraccionarios de la Constante de AMBARD, como en la escala que corresponde al Indice de Mc LEAN, se dificulta la apreciacion del valor funcional de los riñones. Tal es el caso 
cuando se dice que la constante $\mathrm{K}$ es 0,282 , ó, si se expresa que el Indice de Mc. LEAN es 400 o bien 7 .

El concepto Indice Delta.-Determinado como es el nivel normal de funcionamiento renal si se traza una linea en ese punto, es logico considerar que habiendo una alteración que se traduzea por exeso o por deficiencia de trabajo renal, los numeros que marquen exeso sean positivos y los numeros indicadores de deficiencia sean negativos.

Tratandose de un caso cualquiera, tambien es lógico considerar que para referirse a la linea normal, se tenga que expresar con cuantas unidades de exeso o de deficiencia funcionan los riñones y para eso se tiene que comenzar a contar por la unidad.

En resumen se dá un sentido algébrico a la funcion renal, pues, ella cuando alterada como toda funcion-sea ella biologica o matemática-se inclina para mas o para menos. Fuera de esas consideraciones se debe recordar que el Indice de Mc. LEAN, bien interpretado, expresa el porcentaje del valor funcional (yá establecido por CARRION y HALLION, para el caso de que la constante normal se considera 0,070 ) yá que su formula se deduce de la siguiente proporcion: (K normal) ${ }^{2}: 100::$ (K arrormal) $^{2}$ : I en que $K$ es simbolo de la Constante ureo-secretoria de AMBARD é I es simbolo del Indice de Mc LEAN. En la escala construida para expresar el Indice Delta, el cero (0) se corresponde con 0,080 de la Constante de AMBARD y con 100 del Indice de Mc. LEAN. De ese punto cero parten cifras sucesivas en serie ascendente precedidas del signo positivo y cifras en serie descendente precedidas del signo negativo.- Ya se ha expresado que esas cifras representan el porcentage de funcionamiento renal para la excresión de la urea.

Se citan los siguientes ejemplos para hacer ver hasta que punto facilita la apreciaciacion del valor funcional a que nos hemos referido.

Si el Indice Delta en un caso dado es menos treinta por ciento( -30 por ciento), indica que los riñones tinen un valor funcional disminuido en un treinta por ciento o sea que les queda todavia un 70 p. c. de trabajo util. Indice Delta: menos 80 p. c., que apenas los riñones tienen un trabajo util de 20 p. c. Indice Delta: mas 200 o 300 p. c. indican que el v'́lor funcional se ha elevado al doble o al triple de la funcion normal.

Para allanar el segundo inconveniente que se ha citado al comenzar esta exposicion (me refiero al problema numérico que encierra la investigacion del valor funcional de les riñones para la escresion ureica), se ha construido un aparato con la forma y funcionamiento semejantes a los de las reglas de cálculo usuales y que se ha denominado: Aparato para la determinaclon del Indice Delta (cuyo diseño se acompaña en la pag. 15).

No parece indispensable-dada la naturaleza de este trabajo-hacer la descripcion de ese dispositivo mecanico qne reduce el problema numérico a simples manipulaciones de escalas deslizables en fase de otras escalas fijas.

Se considera en cambio necesario hacer presente :

10 - Que el aparato en cuestion determina con mucha exactitud y en el espacio de 30 a 40 segundos, no solo el Indice Delta, que se ha definido, sino tambien, simultaneamente, la Constante ureo-secretoria de AMBARD y el Indice de Mc LEAN. 
20 -Que las escalas $4,5,10$ y 11 no constituyen en si mismas mas que simples escalas de referencia y que tienen como unica función determinar alturas o niveles de las otras escalas que llevan los datos esenciales de los problemas a solucionar.

3o-Las escalas 1, 2, 3, 6 y 9 son destinadas a anotar simplemente los datos proporcionados por el laboratorio, auxiliandose, con algunos puntos de referencia constituidos por saetas.

40 - Las escalas 7 y 8 proporcionan por simple lectura las incognitas buscadas.

Las esplicaciones anteriores tienen el fin de advertir que la impresion de complejidad que queda despues de una simple inspección del conjunto de las escalas, es unicamente aparente, pues se desvanece esa percepcion despues de la verificacion de un problema cualquiera en una primera vez, siguiendo las instrucciones que el aparato lleva en la columna "Manejo".

Cabe todavia hacer una referencia esplicativa relacionada con las formulas, que presenta el aparato en el espacio queda por encima de las escalas 7 y 8 .-

En la determinación de la Constante de AMBARD se contempla dos casos (1): caso en que el problema lleva el factor peso del paciente; (2), caso en que el problema no tiene ese factor. Cada uno de ellos una formula especial:

(1) $\mathrm{K}=\frac{\mathrm{Ur}}{\sqrt{\mathrm{D} \cdot \frac{70}{\mathrm{P}} \times \sqrt{\frac{\mathrm{C}}{25}}}}$

$$
K=\frac{U r}{\sqrt{D \times \sqrt{\frac{C}{25}}}}
$$

Los simbolos que representan los factores que entran en el problema son los siguientes:

(V)-Volumen de la orina recogida.

(C)-Concentración de urea en la orina, gramos por mil.

:T)-Duración de la prueba.

(P)-Peso del paciente.

(Ur)-Concentración de urea en la sangre, gramos por mil.

(K)-Representa la incognita buscada.

En las formulas trascritas anteriormente encontramos que D (debit), representa un valor resultante de calculos previos, efectuados con los valores (C) y (V) en función del tiempo (T). El simbolo D es igual a : $\frac{1440 . \mathrm{V} \text {. C }}{1000 \text {. T }}$

Substituyendo en las formulas citadas en lugar de D sus valores correspondientes tenemos:

(1)

$$
\mathrm{K}=\frac{\mathrm{Ur}}{\frac{\mathrm{P}_{\text {P.1440. V.C } 1000.5} \mathrm{C}}{\mathrm{C}}}
$$

$$
\mathrm{K}=\frac{\mathrm{Ur}}{\frac{\sqrt{1440 \mathrm{~V} . \mathrm{C} V \overline{\mathrm{C}}}}{1.000 \times \mathrm{T} \times 5}}
$$


Simplificando esas formulas y aplicando los logaritmos tenemos:

(1)

$$
K=\frac{U r}{\frac{\sqrt{20,16 . V \cdot C} \bar{C}}{P \times T}}
$$

(2)

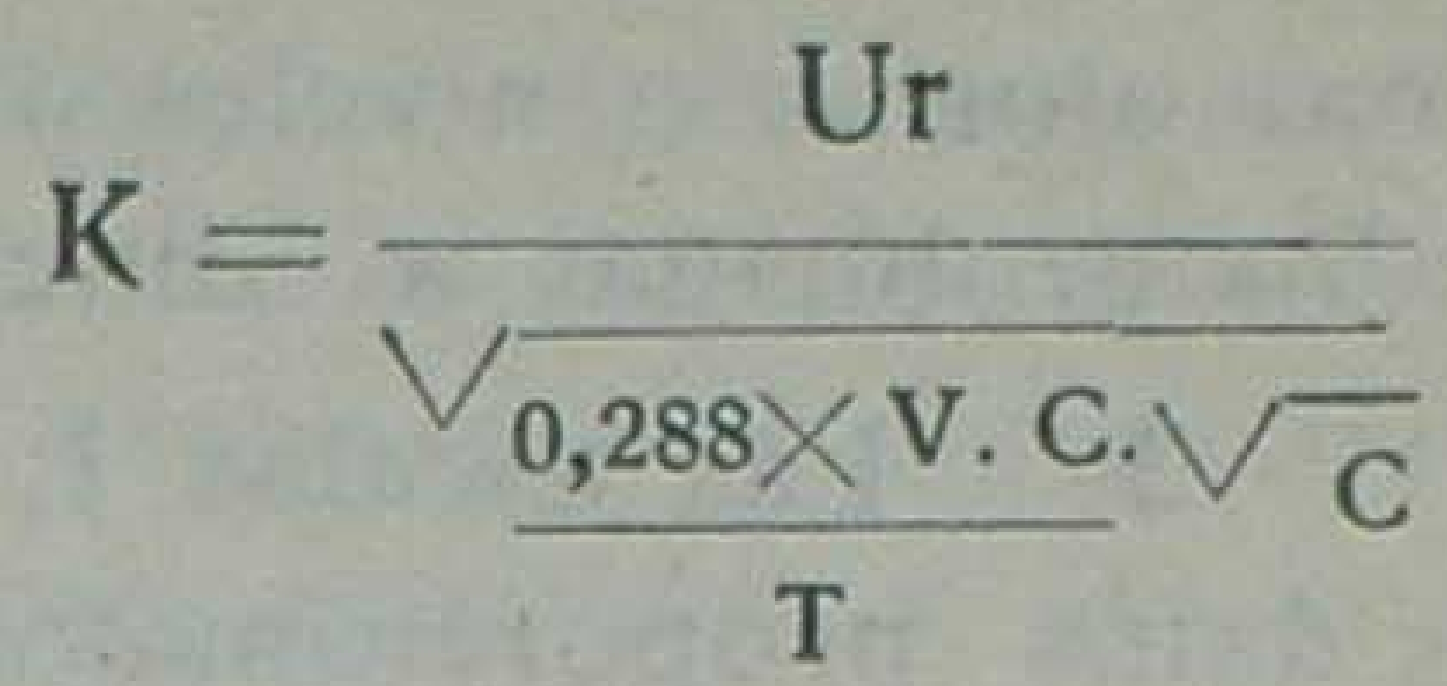
$(\log . \mathrm{P}+\log . \mathrm{T})]$.

(1) $\log . \mathrm{K}=\log \cdot \mathrm{Ur}-1 / 2[(\log \cdot 20,16+\log \cdot \mathrm{V}+3 / 2 \log . \mathrm{C})-$

(2) $\log . \mathrm{K}=\log \cdot \mathrm{Ur}-1 / 2$ I $(\log .0,288+\log . \mathrm{V}+3 / 2 \log . \mathrm{C})-$ log. T) 1 .

Estas ultimas formulas son empleadas en este Instituto, por el Profesor CARNEIRO FELIPPE.

La formula de Mc LEAN, para determinar el Indice de escresion ureica, y que hoy se denomina simplemente Indice de Mc LEAN, es la siguiente: $\quad I=\frac{8,96 . \mathrm{D} \cdot \sqrt{\mathrm{C}}}{\mathrm{P} .(\mathrm{Ur})^{2}}$

Substituyendo D por su valor $\frac{1440 \mathrm{~V} . \mathrm{C}}{1000 . \mathrm{T}}$ y simplificando al formula se tiene: $\mathrm{I}=12,90 . \frac{\mathrm{V} \cdot \mathrm{V} \overline{\mathrm{C}}}{\mathrm{T} . \mathrm{P} \cdot(\mathrm{Ur})^{2}}$

Aplicando logaritmos se tiene: $\log . \mathrm{I}=(\log .12,90+\log . \mathrm{V}+3 / 2$ log. C) $-(\log . \mathrm{T}+\log \cdot \mathrm{P}+2 \log$. Ur).

Esta ultima formula es la que usó el Dr. CARNEIRO FELEPPE en las aulas del Curso.

Conocido el Indice de Mc LEAN, el Indice Delta se obtiene por simple calculo mental, o bien por la formula: $( \pm \delta)=I-100$ y reciprocamente $\mathrm{I}=\delta+100$

En el aparato que ahora se presenta, la escala, 8, traduce al mismo tiempo, el Indice de Mc LEAN y el Indice Delta. Basta suponer en esa escala anotados los numeros de orden en serie ascendente comenzando por la unidad constituyendo asi la escala Indice de Mc LEAN, en correspondencia de la escala del Indice Delta.

En resumen este trabajo presenta a la consideración de los clinicos y laboratoristas :

10-El concepto Indice Delta, que es la tradución al lenguaje matemático de un fenomeno biológico.

2o-Un aparato, que por simples manipulaciones, determina no solo el Indice Delta que se ha definido, sino tambien y simultaneamente, el Indice de Mc LEAN y la Conslante ureo secretoria de AMBARD. 

CUADRO COMPARATIVO DE LAS RELACIONES EXISTENTES ENTRE LOS TRES SISTEMAS: CONSTANTE DE AMBARD, INDICE DE MC LEAN E INDICE DELTA.

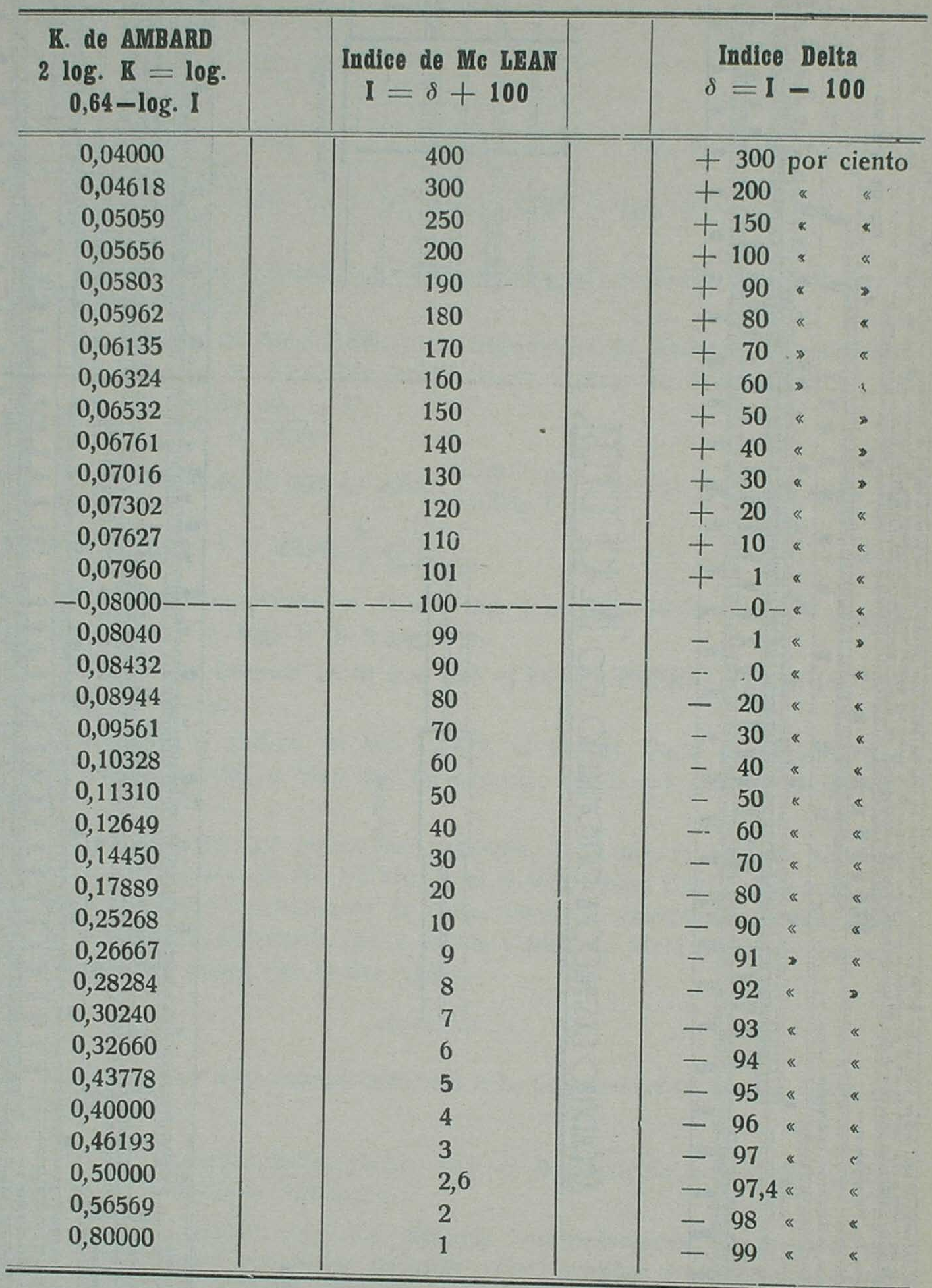

NOTA:-Para buscar el Indice de Mc LEAN, conocida la Constante de AMBARD, la formula es: $\log$. $\mathrm{I}=\log .0,64-2 \log$. $\mathrm{K}$. 